\title{
Einige Erinnerungen an meine Zeit an der Augenklinik
}

\author{
Christian Skorpik
}

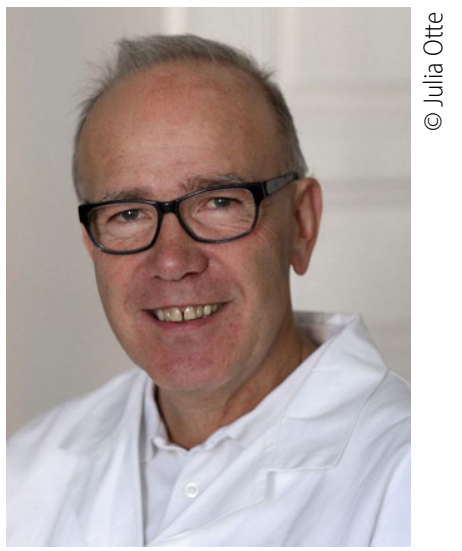

Als 1987 das erste Heft spektrum der augenheilkunde erschien, war ich bereits das 8. Jahr an der ersten Universitäts-Augenklinik tätig. Es war uns allen, insbesondere aber unserem damaligen Klinikvorstand, Univ.Prof. Dr. Heinz Freyler, ein großes Anliegen, das Erscheinen der Zeitschrift zu ermöglichen, und wir bemühten uns daher auch, durch möglichst gehaltvolle Beiträge, die Qualität der neuen Fachzeitschrift zu dokumentieren.

Im Januar 1980 war ich als Assistenzarzt an die erste Universitäts-Augenklinik gekommen - damals unter der Leitung von Univ.-Prof. Dr. Karl Hruby. Relativ bald durfte ich Prof. Hruby in seiner Privatordination assistieren und konnte dadurch einen guten Einblick in die Arbeitsabläufe in einer Ordination gewinnen. Das hat mir später in meiner beruflichen Tätigkeit sehr geholfen. Es war doch ein ganz anderes Arbeiten als in der Ambulanz der Augenklinik. Damals musste jeder

Univ.-Prof. Dr. C. Skorpik $(\bowtie)$

Universitätsklinik für Augenheilkunde und Optometrie, Medizinische Universität Wien, Währinger Gürtel 18-20, 1090 Wien, Österreich

christian.skorpik@meduniwien.ac.at
Patient in der Ambulanz möglichst komplett durchuntersucht werden, was meist sehr zeitaufwendig war. Das ist nicht mit den heutigen Ambulanzabläufen, die schnell und lösungsorientiert sein müssen, vergleichbar. Der Lerneffekt war aber sehr groß. Ich hatte hervorragende Lehrer und Vorbilder, insbesondere Hans Gnad und Susanne Binder möchte ich hervorheben.

Als ich in der ersten Universitäts-Augenklinik begann, wurde die Kataraktoperation noch als ICCE (Intrakapsuläre Kataraktextraktion) durchgeführt, meist ohne Kunstlinsenimplantation. Immer häufiger jedoch erfolgte die Implantation von Kunstlinsen. Dabei wurden an unserer Klinik vor allem 4-Schlingen-Linsen nach Binkhorst, aber auch kammerwinkelgestützte Vorderkammerlinsen eingesetzt. 1984 veröffentlichte ich z. B. eine Serie von Vorderkammerlinsenimplantationen mit der sogenannten „Shepard-Vorderkammerlinse“. Damals wurde bereits mit dem „Klöti-Stripper“ oder dem „Ocutome nach O'Malley“ gearbeitet, um vorgefallenen Vitreus zu entfernen. Diese Linse, wie auch andere Vorderkammerlinsen, galten schon damals an unserer Klinik als Kompromissimplantat bei Kapselruptur, denn routinemäßig wurde damals bereits seit 1982 die ECCE (Extrakapsuläre Kataraktextraktion) mit Hinterkammerlinsenimplantation (Simcoe C-loop Hinterkammerlinsen mit $10^{\circ}$ Schlaufenneigung) durchgeführt. Die Schlaufenneigung bewährte sich damals schon um Irisschädigungen sowie „pupillary capture“ bei Mydriasis zu verhindern. Es gab ja zu der Zeit noch keine Kapsulorhexis, sondern erst die „can-opener Kapselöffnungstechnik“. Dabei war der Linsensitz nicht immer eindeutig ,in the bag“. Die ECCE-Ära hatte mit der Einführung des „Klöti-Strippers“ begonnen, den Hans Gnad aus seiner Zeit in Zürich mitbrachte und bei uns unter anderem zum Absaugen von Linsenkortexmaterial etablierte. Anfangs wurden kurzzeitig auch bei ECCE 4-Schlingen-Lin- 
sen nach Binkhorst implantiert. Dabei kam es jedoch postoperativ oft zu Dezentrierungen, obwohl die Kapsel mit dem Vitreus-Stripper nachgeschnitten werden konnte. Die Ergebnisse mit der seit 1982 implantierten Hinterkammerlinse nach Simcoe waren eindeutig überlegen, daher wurden diese „Three Piece Linsen“ bevorzugt verwendet. 1984 wurde an unserer Klinik die Phakoemulsifikation etabliert, und ich hatte das große Glück, bei diesen Entwicklungen von Anfang an dabei sein zu dürfen. Durch diese neue Technik konnten dank des kleinen Schnittes auch kombinierte Operationstechniken, z. B. Kataraktoperation und IOL Implantation kombiniert mit Trabekulektomie, erfolgreich angewendet werden.

Parallel dazu wurde 1983 an unserer Klinik von Rupert Menapace und von mir die Glaukomambulanz gegründet, die ich bis 1986 leitete. In dieser Zeit wurde auch die Argonlasertrabekuloplastik als wenig invasiver Eingriff eingeführt. 1985 wurde ich als Oberarzt mit der Leitung einer Station betraut.

Die Kataraktchirurgie war von Anfang an ein Thema, das mich intensiv beschäftigte. Das anfangs verwendete Linsenmaterial war PMMA (Polymethylmethacrylat) mit Prolenebügeln. Es bestand jedoch der Wunsch nach besserer Gewebeverträglichkeit und Linsenimplantation durch eine möglichst kleine Schnittöffnung, was durch die Verwendung der Phakoemulsifikation möglich wurde. Als Material stand damals einerseits Hydrogel und andererseits Silikon zur Verfügung. Rupert Menapace und ich testeten intensiv beide Materialien. Von Vorteil war es, dass es sich dabei um flexible Materialien handelte. Die Linsen konnten dadurch gefaltet durch kleinere Wundöffnungen implantiert werden. 1987 und 1988 implantierten wir unter der Federführung von Rupert Menapace eine Serie von Kunstlinsen aus Hydrogelmaterial (IOGEL PC12 der Firma Alcon) als Kleinschnittchirurgie. 1986 erfolgten die ersten Silikonlinsenimplantationen der Firma STAAR Surgical in den Sulcus. Im August 1987, also gleichsam zum Gründungszeitpunkt des spektrum der augenheilkunde begann ich mit der Implantation von gefalteten Silikonlinsen in den Kapselsack nach Phakoemulsifikation mit dem Gerät Cavitron 9000 der Firma Cooper Vision. Das Thema „Klinische und experimentelle Ergebnisse nach Implantation von Hinterkammerlinsen aus Silikonmaterial" wurde 1988 auch meine Habilitationsschrift, die im spektrum der augenheilkunde als Supplementum III veröffentlicht wurde. Im Dezember 1991 begannen wir torische Hinterkammerlinsen aus Silikonmaterial der Firma STAAR Surgical mittels Kleinschnittchirurgie in den Kapselsack zu implantieren und beobachteten die Rotationsstabilität. Ermöglicht wurde die sichere Implantation der Kunstlinsen in den Kapselsack erst durch die Einführung der Kapsulorhexis-Technik Mitte der 80er-Jahre. Anfang der 90er-Jahre begannen wir unsere Operationstechnik auf großteils „No Stitch Chirurgie“ umzustellen und in weiterer Folge auch die Technik der „Clear Cornea
Incision“ anzuwenden. In diesem Zusammenhang erprobten wir dann erfolgreich die Operationen in Tropfanästhesie mit Lidocain. Ebenfalls in den 90erJahren implantierten wir bereits multifokale Hinterkammerlinsen und zwar anfangs die diffraktive Linse 815LE der Firma 3M und später dann auch die Arraylinse der Firma AMO. Die Biometrie war in den ersten Jahren der Kunstlinsenimplantation auch erst in den Kinderschuhen, und die ersten Linsenimplantationen erfolgten nach Schätzung der Refraktion. Wir bemühten uns sehr früh um möglichst exakte und reproduzierbare Daten für die Linsenstärkeberechnung. Hans Gnad, Panayote Paroussis und ich fuhren nach Zürich und holten die von Christoph Huber 1981 entwickelte Kunstlinsenberechnungsmethode mittels eines Hewlett-Packard ${ }^{\circledR}$-Rechners nach Wien.

Die erste Universitäts-Augenklinik war damals eines der führenden und innovativsten chirurgischen Zentren in Europa und auch im deutschsprachigen Raum. Ermöglicht wurde das letztendlich durch unser Engagement und durch die großartige Unterstützung unseres damaligen Klinikvorstandes Univ.-Prof. Dr. Heinz Freyler. Zur damaligen Zeit existierte an unserer Klinik noch keine Trennung in vorderen und hinteren Augenabschnitt. Als Chirurg war man für alles zuständig. Daraus ergaben sich viele interessante Arbeitsbereiche und Querverbindungen.

Das erste Heft des spektrum der augenheilkunde spiegelt diese Vielfalt wider. Es wurde über Biometrie publiziert, aber auch über Ergebnisse von Vitrektomie bei Ruptur der Linsenkapsel bei Kataraktoperation. Mein späterer chirurgischer Schwerpunkt, die Keratoplastik, war auch damals bereits für mich ein Thema. Ich publizierte eine Arbeit über kombinierte Keratoplastik und Kunstlinsenimplantation, was seit 1983 an unserer Klinik durchgeführt wird.

Während meiner ersten Jahre an der Klinik hatte ich die glückliche Gelegenheit mit Susanne Binder gemeinsam experimentelle Projekte im Bereich des hinteren Augenabschnittes durchführen zu dürfen und von ihr und Hans Gnad in die Vitrektomie eingeführt zu werden. Seit 1982 wurde mit Silikonölimplantation gearbeitet. Die damalige Technik wurde von Hans Gnad während eines Aufenthaltes bei Zivojnovic in Rotterdam erlernt und von unserer Arbeitsgruppe an der ersten Universitäts-Augenklinik übernommen. Dadurch konnte ich sehr früh während meiner klinischen Tätigkeit schon komplexe Ablatiochirurgie und Traumatologie erlernen.

Seit 1988 leitete ich die Hornhautambulanz, die Refraktionsambulanz sowie die Kunstlinsenambulanz. Aus dieser Situation heraus ergab sich für mich die Möglichkeit, bereits 1992 mit einem AesculapMeditec Mel 60 Excimerlaser $\mathrm{zu}$ arbeiten und PRK (Photorefraktive Keratektomie) Behandlungen an der ersten Universitäts-Augenklinik durchzuführen. Die Laserbehandlungen wurden später mit dem Excimerlaser Keratom der Firma Schwind fortgesetzt. Nun ist ein Laser der neuesten Generation im Einsatz. Die 


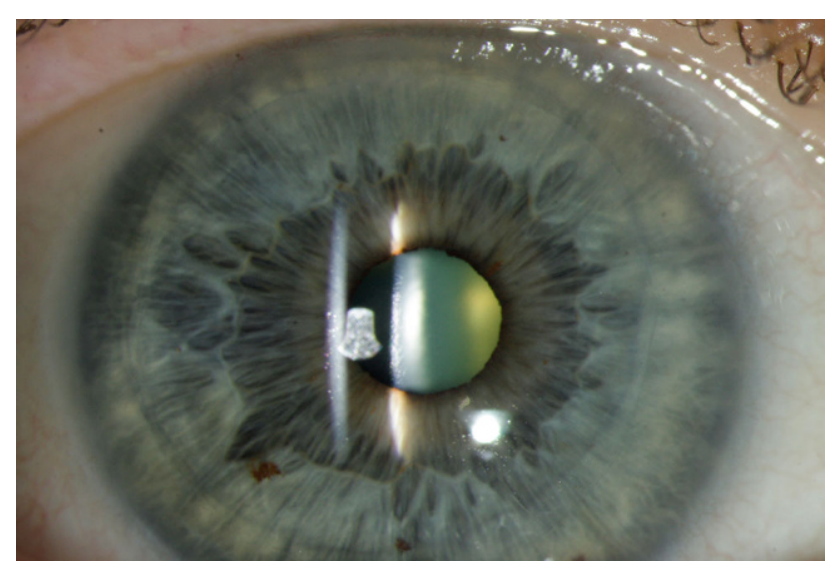

Abb. 1 DS(A)EK

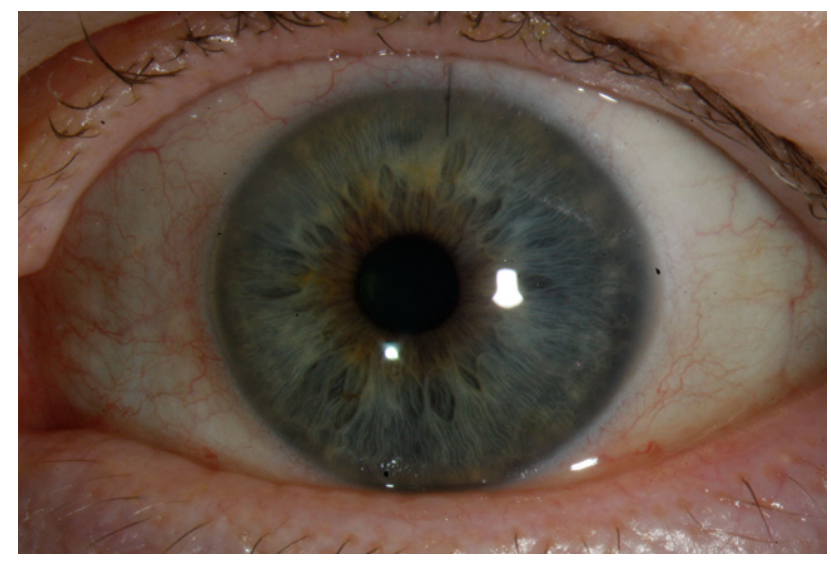

Abb. 2 DMEK

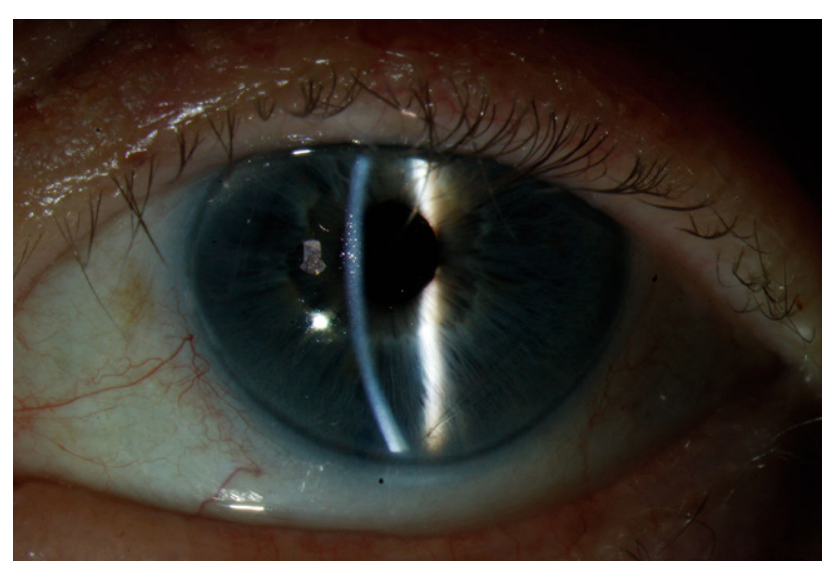

Abb. 3 DMEK Spaltlampenbild mit Transplantatfalten temporal unten

LASIK-Behandlung erfolgte mit einem Mikrokeratom der Firma Gebauer. Die Tradition modernste Methoden zur Behandlung von Fehlsichtigkeit und Hornhautanomalien anzuwenden wird an der UniversitätsAugenklinik in Wien unter der Leitung von Stefan Pieh weitergeführt. Der Femtosekundenlaser der Firma Ziemer ergänzt nun das Instrumentarium. Zur Behandlung hoher Fehlsichtigkeit wurde im September 1993 die ICL $^{\circledR}$ (Implantierbare Kontaktlinse) der
Firma STAAR Surgical an unserer Klinik im Rahmen der Refraktionsambulanz eingeführt. Das Konzept erschien mir vielversprechend und zukunftsträchtig. Ich konnte damit gute bis sehr gute Ergebnisse erzielen. Die einzige relevante Einschränkung während der Langzeitbeobachtung war die Zunahme der Kataraktentwicklung. Diese Anfangsschwierigkeiten scheinen nun dauerhaft behoben $\mathrm{zu}$ sein und die $\mathrm{ICL}^{\circledR}$ ist in modernisierter Form immer noch als „Visian ICL ${ }^{\circledR}$ “ in breiter Verwendung.

Die Hornhaut blieb jedoch im Laufe der Jahre mein Hauptarbeitsgebiet. Anfang 1992 begannen wir Keratoplastiken routinemäßig mit dem GTS (Geführtes Trepansystem) nach Krumeich durchzuführen. Die Ergebnisse damit waren ausgezeichnet und die Technik hat sich viele Jahre gut etabliert gehalten. Erst durch die neueren Entwicklungen mit dem Femtosekundenlaser hat diese Technik ihren hohen Stellenwert verloren. Angeregt durch die Mikrokeratomerfahrungen bei LASIK und Publikationen von Busin begann ich 2002 mit der Endokeratoplastik bei Hornhautendotheldystrophie, wobei die vordere Hornhauthälfte mittels eines partiellen Mikrokeratomschnittes erhalten wird und nur der hintere Hornhautanteil dann durch die „open sky“-Technik ersetzt wird. Die vordere Hornhaut wird wieder zurückgeklappt und mit Nähten fixiert. Zur besseren Adaptation wird kurzzeitig Luft in der Vorderkammer belassen. Die Ergebnisse damit waren gut und funktionell zufriedenstellend. Dank der guten Erfahrungen mit dem dafür verwendeten ALTK (Automated Lamellar Therapeutic Keratoplasty) Mikrokeratomsystem der Firma Moria war es nur ein kleiner Schritt 2006 auf die DS(A)EK (Descemet's Stripping [Automated] Endothelial Keratoplasty)Technik umzusteigen. Mit dieser nahezu nahtlosen Technik wurden die Behandlungsergebnisse noch weiter verbessert. Es gab nur mehr kleine Einschnitte und die Architektur der Hornhaut blieb weitgehend unbeeinflusst. Die Hornhaut wird nur etwas dicker, meist um $100 \mu$ bis $130 \mu$. Das operationstechnisch bedingte Meniskusdesign des Transplantates macht insbesondere bei dickeren Transplantaten die Zentrierung wichtig, da es bei Exzentrizität leicht zu optischen Aberrationen kommen kann. Die Methode hat sich bis heute in verschiedensten Modifikationen sehr bewährt und ist für viele Chirurgen noch heute die bevorzugte Technik (Abb. 1). Der Schnitt kann nun auch mit einem Femtosekundenlaser durchgeführt werden, wodurch dünnere Transplantate mit exakt vorherbestimmbarer Hornhautdicke und ohne Meniskusform möglich sind. Die Entwicklung ging jedoch wieder weiter und wir starteten 2012 mit der Descemet-MembranTransplantation, der DMEK (Descemet Membrane Endothelial Keratoplasty). Diese Operationstechnik wird zurzeit von Gerald Schmidinger und von mir am AKH Wien durchgeführt und damit lassen sich die Ergebnisse in Bezug auf die DS(A)EK noch weiter verbessern (Abb. 2 und 3). Die DMEK ist nun an unserer 


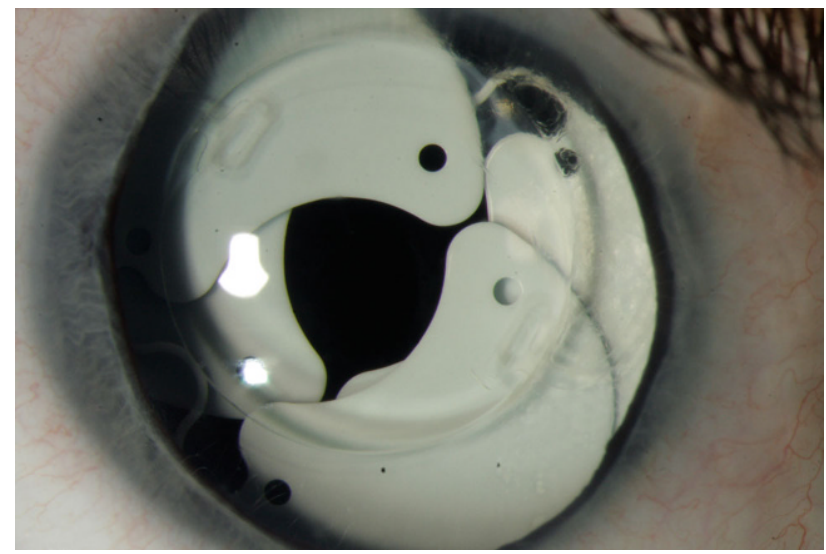

Abb. 4 Oculaid ${ }^{\circledR}$ Irissektorimplantate der Firma OPHTEC und IOL im Kapselsack bei traumatischer Iridoplegie

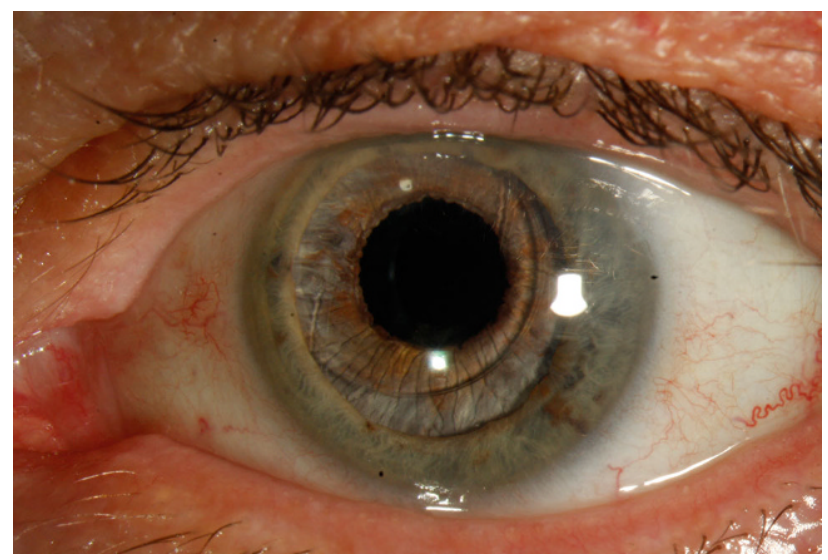

Abb. 5 Artificial Iris ${ }^{\circledR}$ und IOL

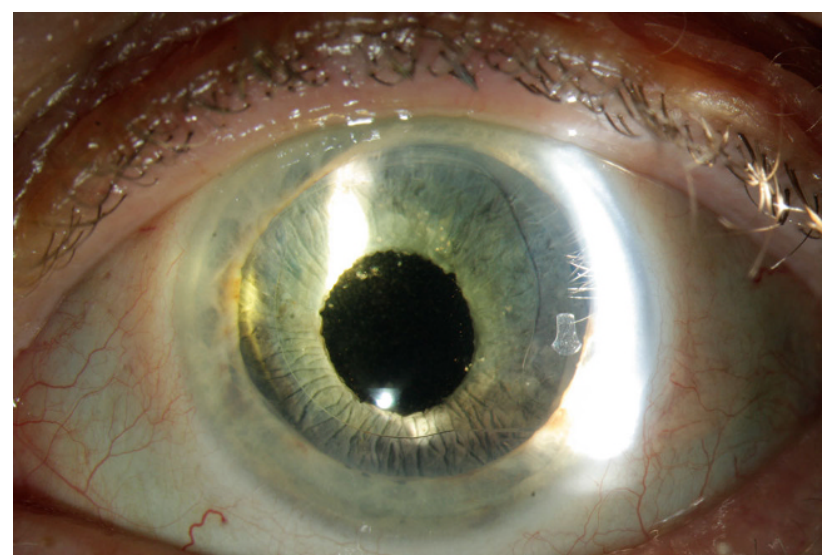

Abb. 6 Artificial Iris ${ }^{\circledR}$ und IOL

Klinik die bevorzugte Operationstechnik bei Endothelversagen der Hornhaut und eine DS(A)EK wird nur mehr extrem selten durchgeführt. Die Operation selbst ist wiederum um einiges anspruchsvoller als die DS(A)EK und die Lernkurve auch entsprechend länger. Es gibt mittlerweile verschiedenste Modifikationen der Technik. Einer der großen Vorteile gegenüber der perforierenden Keratoplastik ist die meist problemlose Wiederholbarkeit der Operation (Austausch



Abb. 7 Artificial Iris ${ }^{\circledR}$ und Hornhautnarben nach Verletzung

der Descemet-Membran und des Endothels) ohne die restliche Hornhaut nachhaltig zu beeinträchtigen. Die perforierenden und vorderen lamellären Keratoplastiken werden nun meist mit dem Femtosekundenlaser durchgeführt. 2012 begannen wir bei der vorderen lamellären Keratoplastik (DALK, Deep Anterior Lamellar Keratoplasty) auch mit der sogenannten „ big bubble“-Technik. Auch diese Operationstechnik hat sich sehr bewährt und wird an unserer Klinik derzeit bei entsprechender Indikation routinemäßig angewendet. So geht die Entwicklung erfreulicherweise immer weiter, und bei Keratokonus wird an unserer Klinik seit Jahren von Gerald Schmidinger und Mitarbeitern „Corneal Cross Linking“ mit großem Erfolg durchgeführt. Auch werden Ringsegmente eingesetzt, um die Hornhautkrümmung zu verbessern. Mit dem Excimerlaser wird die Hornhautoberfläche nach Keratoplastik bei Bedarf optimiert und reguläre sowie auch irreguläre Astigmatismen werden reduziert. Das ist im Rahmen der Refraktionsambulanz einer der Arbeitsschwerpunkte von Stefan Pieh.

Traumatologie und rekonstruktive Chirurgie waren mir von Anfang an immer wichtig. Dabei müssen oft komplexe Behandlungsstrategien angewendet werden. Bei Verletzungen oder Erkrankungen mit Irisschädigung implantierten wir früher immer wieder Spannringe mit Irissegmenten, sulcusgenähte Kunstlinsen mit Iriszeichnung sowie Irissegmente in den Kapselsack (Abb. 4). Im Gegensatz zu diesen starren Implantaten mit nur wenigen Farbvarianten, welche eine große Wundöffnung benötigen, verwenden wir seit einigen Jahren die Artificial Iris ${ }^{\circledR}$ der Firma HumanOptics AG, welche gefaltet über einen relativ kleinen Schnitt implantiert werden kann und entweder in den Kapselsack gegeben, in den Sulcus gesteckt oder genäht wird. Dieses großteils aus Silikonmaterial gefertigte Implantat kann auch zurecht geschnitten werden und nur in Teilen implantiert werden. Die Irisfarbe wird individuell von der Firma produziert und an das Partnerauge angeglichen (Abb. 5, 6, 7 und 8).

Als Folge der immer älter werdenden Bevölkerung kommt es öfter als früher zu Kunstlinsensubluxatio- 


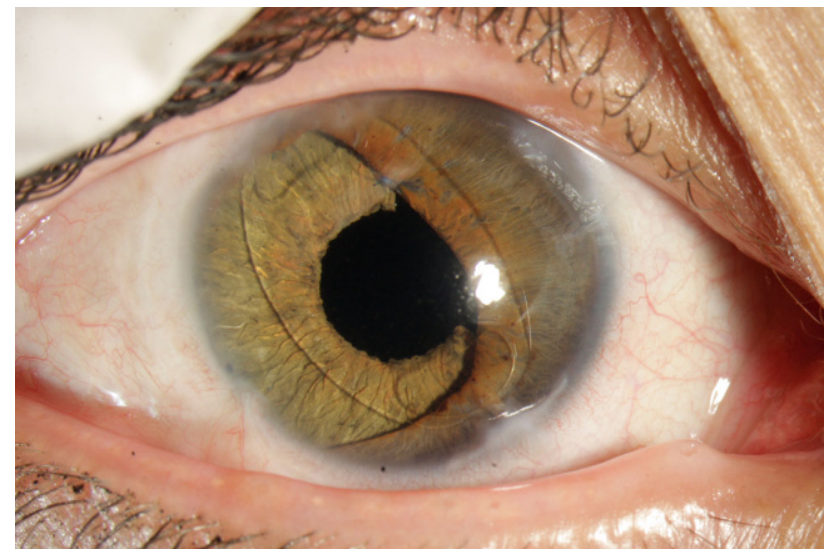

Abb. 8 Artificial Iris ${ }^{\circledR}$ Teilimplantat und Artisan ${ }^{\circledR}$ IOL

nen oder Luxationen. Die Ursachen sind vielfältig und nicht nur Augen mit Pseudoexfoliationssyndrom sind davon betroffen. Verschiedenste Lösungsansätze sind bei einer solchen Problemstellung möglich und in Erwägung zu ziehen: Refixation der Linse, Spannringfixation oder Kunstlinsentausch mit oder ohne Vitrektomie. Diese Operationsindikation wird in Zukunft noch beträchtlich zunehmen.
Erwartungsgemäß hat sich in den letzten 37 Jahren, seit ich an der Augenklinik bin, vieles weiterentwickelt und es ergeben sich immer wieder neue Fragestellungen und neue Therapiekonzepte. In den 30 Jahren, seit es das spektrum der augenheilkunde gibt, hat vieles davon auch seinen Niederschlag in der Zeitschrift gefunden. In vielen Bereichen der Augenheilkunde gehen die Wurzeln für die derzeitigen Standards weit zurück und vieles war früher bereits ansatzweise vorhanden und möglich. Doch die Verbesserungen und die Qualitätszunahme sind gewaltig. Insbesondere haben bildgebende Verfahren wie OCT, computergesteuerte Bildauswertung oder Gewebeanalysesysteme viel zur Weiterentwicklung beigetragen. In Zukunft werden auch viele weitere Entwicklungen wie Robotik, optimierte Netzhautimplantate etc. die Ophthalmologen herausfordern. Aber auch interdisziplinäre $\mathrm{Zu}$ sammenarbeit wird einen immer höheren Stellenwert einnehmen. Wie in anderen Disziplinen auch müssen immer wieder neue, noch unbekannte Pfade angelegt und begangen werden. Man muss immer wieder ehrgeizig nach Neuem suchen, um Wissen und Können zu erweitern.

Interessenkonflikt C. Skorpik gibt an, dass kein Interessenkonflikt besteht. 